\title{
Integration of Information for Patient Care: 2015 Redux
}

\author{
0. Rienhoff \\ Institut für Medizinische Informatik, Universitätsmedizin Göttingen, Göttingen, Germany
}

In 1996, the IMIA Yearbook of Medical Informatics reflected on the long-term technical and methodological developments in medical and health informatics, which had led eventually to very powerful integrated workstations for healthcare professionals. Although this topic had already been discussed for about ten years in 1996, the impression was then that the computing power, the screen display resolution, and the communication speed had reached a sufficient quality and cost-efficiency to allow for integrated workstations, including image processing, available at reasonable cost. Thus, the expectation in 1996 was that it had become feasible to bring onto the desk of health-care professionals, a multiplicity of computer-supported functions that would change the way these professionals would handle patient information. Even more important, it was seemed to be possible not only to have multimedia processes but also to link operational systems to various kinds of knowledge bases and thus to directly support clinical decision-making as part of the therapeutic management of patients.

In 2016, some of the technical aspects of the 1996 preface are antique - however the key message has remained true. The technical development in biomedical informatics has moved ahead but the gap between technology and operational use in health care is still an issue. Although leading wealthy institutions are further down the operational path, the gap between potential opportunities and everyday reality is a reality of our field. This reality is typical of all fast moving, technologically-empowered businesses such as science and healthcare, and it is true for the ongoing development in biomedical informatics. The various IMIA

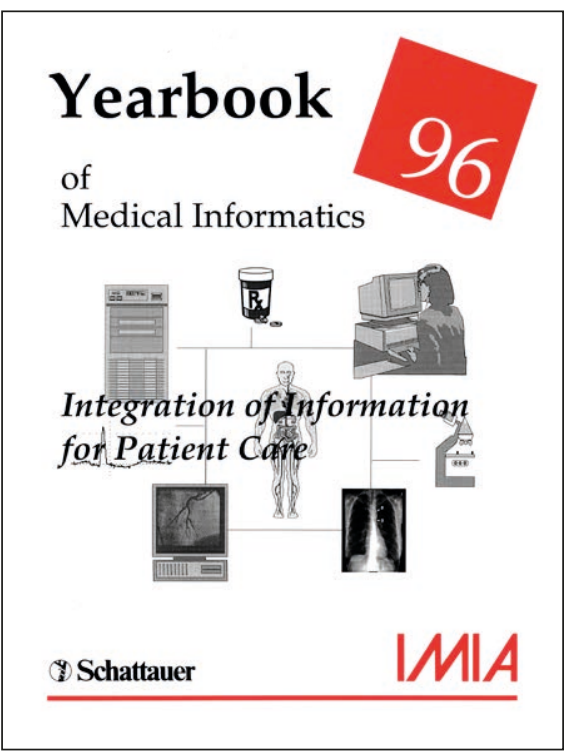

member countries have struggled with this gap - with smaller countries leading the development. Thus, the preface to the 1996 IMIA Yearbook can be left as it was written - just some sentences may need to be updated with new examples.

The question of how to design powerful integrated workstations for healthcare professionals is closely related to several methodological questions, of which the following two seem to be the most important ones:

1. How to define the architecture of an electronic patient record?

2. How to link decision-support, algorithms, and knowledge from medical databases to the everyday workflow?

Local legal requirements, differences among national medical and social systems, and specificity of clinical and ambulatory settings will lead to very different designs for electronic patient records according to the countries or sub-sectors within national health-care systems. The question of whether and how managed care approaches are implemented has a particularly major influence on the architecture of the electronic patient record. It will require major efforts of healthcare informatics specialists in many countries to finally reach a consensus and decide whether the various considerations can be mapped into a general architecture, or if different 
types of architecture should be developed, programmed, and maintained.

The linkage of relevant information and knowledge for the support of decision processes in the everyday work of healthcare professionals is the second major topic to be addressed. All over the world, more and more commercial companies are entering the expanding market to provide and to sell specialized information services to health-care professionals. The dynamic development of this sector is closely related to the expectations of several international companies that consider that their future customers are not only health care providers, but rather the average citizen, who is interested in learning more about her/his health - a notion addressed in 2016 through the complex world of pa- tient-based health sensors. A huge market is anticipated and major investments are being directed into the development of corresponding databases and on-line services. The impact that this development may have on the health professional's workstation and the workflow may be considerable. It may well be that the Quantified Self movement will change not only workflow patterns in healthcare, but also provider-patient relationships, organizational hierarchies, and the overall system of how knowledge is handled in health-care systems, science, and research. Therefore, research on the health-professional's workstation must not only have a technical dimension, but also requires addressing the social and organizational consequences that those technical changes may bring.
When the first computers became available in the forties and fifties of the $20^{\text {th }}$ century, applications in healthcare were immediately discussed. Since then, many Medical Informatics specialists have contributed to the advancement of the field. The IMIA Yearbook has been documenting and portraying this development, and, as we see it today, it addressed many issues long before it was clear that they would accompany the historical development as a constantly reshaping set of methodological challenges.

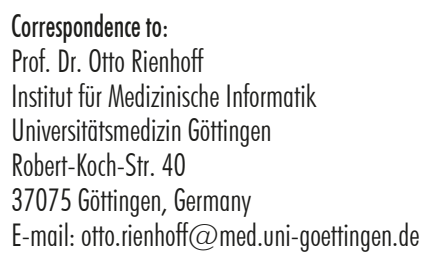

